\title{
Impact of Artificial Intelligence-enabled Software- defined Networks in Infrastructure and Operations: Trends and Challenges
}

\author{
Mohammad Riyaz Belgaum ${ }^{1}$, Shahrulniza Musa ${ }^{3}$ * \\ Malaysian Institute of Information Technology \\ Universiti Kuala Lumpur \\ Kuala Lumpur, Malaysia \\ Zainab Alansari ${ }^{2}$ \\ Faculty of Information Technology \\ Majan University College, Muscat, Oman \\ University of Malaya, Kuala Lumpur, Malaysia
}

\author{
Muhammad Mansoor Alam ${ }^{4}$ \\ Malaysian Institute of Information Technology \\ Universiti Kuala Lumpur. Kuala Lumpur, Malaysia \\ Institute of Business Management, Karachi, Pakistan
}

\begin{abstract}
The emerging technologies trending up in information and communication technology are tuning the enterprises for betterment. The existing infrastructure and operations (I\&O) are supporting enterprises with their services and functionalities, considering the diverse requirements of the end-users. However, they are not free of the challenges and issues to address as the technology has advanced. This paper explains the impact of artificial intelligence (AI) in the enterprises using software-defined networking (SDN) in I\&O. The fusion of artificial intelligence with software-defined networking in infrastructure and operations enables to automate the process based on experience and provides opportunities to the management to make quick decisions. But this fusion has many challenges to be addressed. This research aimed to discuss the trends and challenges impacting infrastructure and operations, and the role of AI-enabled SDN in I\&O and discusses the benefits it provides that influence the directional path. Furthermore, the challenges to be addressed in implementing the AI-enabled SDN in I\&O shows future directions to explore.
\end{abstract}

Keywords-Artificial intelligence; infrastructure and operations; software-defined network; virtualization

\section{INTRODUCTION}

Industry 4.0 puts together dynamic systems, which exist in several conflicting ways in their practical implementation within businesses. "4.0" is far from being a paradigm but composite geography which differentiates itself from the management culture, organizational growth, technical choices, the position of employees, regulatory structures, and the expectations of labour unions. Inside factories, it is the mixture of the physical world between manufacturing processes and the fictional environment of digital knowledge. Within the enterprise, the infrastructure and operations are adopting intelligent technologies in line with the vision of industry 4.0 [1]. The enabling technologies allowing for the smart environment are the Internet of Things (IoT), 5G networks [2], big data, cloud computing, virtual and augmented reality, artificial intelligence, and multi-access edge computing [3] skills. Many new security and networking problems continue to emerge as IT systems are developed with these technologies. While these technologies have clear benefits, the ability of an organisation to support and sustain quick, high-demand supply chain operations is not therefore sufficient if traditional network technology is considered. Networking is the cornerstone of business growth in today's ever-connected world. For regular those complex supply-chain activities, producers, manufacturers, distributors, and consumers must be dynamic as company branches that rely on immediate communications for their offices. This is necessary if the supply chain is to have the needed management versatility and visibility. IoT devices, sensor connectivity, and other cyber-oriented systems are expanding, fostering development at the edge and on the network. The transformation from conventional networking to SDN brings efficiency, agility, and reduces costs [4].

Software-defined networking (SDN) is a modern paradigm in the field of networking, making it more agile by programming the network. This feature of customizing the network enables the infrastructure and operations to utilize better the resources based on the needs of enterprise and market demand. The end-user devices, storage data centres, and wide area networks are the players moving in a softwaredefined industry which enhances the control, programming, and response to business requirements of network operators. The motivation behind the move to SDN and Network Functions Virtualizations (NFV) has led to software and virtualization taking advantage that is based on agility, flexibility, and adaptability. This changes considerably the way networks are designed, managed and services delivered. Software, which becomes an increasingly important part of every network, embraces changing end-user criteria for greater programming and transparency. This level of business change requires significant restructuring of the network. Softwaredefined wide area networks [5] (SD-WANs) with a significant focus on SD security with many software platforms of

*Corresponding Author 
companies is one of the most effective and efficient ways of achieving this milestone. The combination of various systems allows the manufacturers to operate more efficient, fast, and cost-effectively, while rising bandwidth. Also, synergies between SD-WAN and the multi-cloud architectures [6] offer several advantages to automation and virtualization and ondemand provisioning, while maintaining mobility and cost savings.

New technology deployment is not risk-free, and a company needs to evaluate its strategies for mitigating any threats to business continuity. To know where the threat comes from, first and foremost, it is essential to understand that any interruption to the company, regardless of how complicated, can be detrimental. Instead of a separate transportation line for enterprise-related networking, the use of cloud and SaaS can substitute the same reducing the security vulnerabilities [7]. Finally, it is essential to acknowledge that real innovation is not inherently the responsibility of existing vendors who have a crucial involvement in most companies. Organizations must also consider and depend on start-up innovation to take advantage of innovative solutions. Nevertheless, not all obstacles may be faced by SD-WAN. For instance, if there is a small business that only has one website, there is no need for SD-WAN if only a limited number of applications rely upon it. Moreover, when using the internet, it is still required to protect how the offices connect to the cloud or SaaS, consolidating multiple devices on the branch to create the software-defined branch. Currently, most organizations are using traditional networking and are ending up incurring huge expenses on administrating the network management. Realizing the benefits of SDN they are transforming towards SDN to reduce the CAPEX and OPEX. The fusion of AI into SDN makes the process automated without human intervention. The basic purpose of this study is to explore some challenges faced in artificial intelligence-enabled SDN in $\mathrm{I} \& \mathrm{O}$ with a focus on the following.

- Trends impacting the I\&O which play a significant role and are expected to accompany the commitments of organizations are discussed.

- The impact of AI on I\&O with AI-enabled SDN in I\&O along with the benefits are also discussed.

- Stepwise process of transforming a commoditized AI algorithm to a customized AI algorithm is presented.

- Challenges and future directions arising in merging these technologies.

The remaining paper is structured in the following way. Section 2 presents the related literature. Section 3 has a discussion part with the trends impacting I\&O and the impact of AI on I\&O. The challenges and future directions are presented in Section 4. In Section 5, the conclusion is presented.

\section{RELATED LiTERATURE}

AI is a game-changing technology, which is integrated with various other technologies to give optimized results. In this section, some of the related studies have been discussed, which shows how AI diffusion has impacted in improving the performance of different techniques in different domains.

Blockchain is a distributed technology that is currently being used to ensure secure and irreversible transactions. The authors in [8] have proposed a data-sharing mechanism with a focus on the network operations happening without human intervention. A trusted data-sharing framework was presented by designing a smart contract using DataChain and BehaviorChain. The smart contract grants permission to access considering the access methods and requirements. In [9], the authors have used AI in civil infrastructures to assess the visual quality of the constructions made. The authors proposed a smart mixed reality framework, by integrating it with a wearable device to detect the cracks. The mixed reality technology will help the investigators to assess and take decisions by improving the visual inspection.

Operations management $(\mathrm{OM})$ is a field of management that converts raw materials and labour into products and services. The authors in [10] studied the feasibility of AI in OM considering various factors and proposed guidelines for the managers to take decisions. The results of the study present that the utilization of AI in OM increases efficiency, quality, and customer satisfaction. Also, they conclude that the adoption of $\mathrm{AI}$ in $\mathrm{OM}$ can be successful when the human and AI both function symbiotically. The digital transformation strategy (DTS) discussed in [11] is in a continually evolving state. A framework to transforms from pre-digital organizations to a fully digital system has been proposed. A thorough study of this transformation has been discussed in a phase-wise manner until it is realized that DTS has been attained. The authors in [12] briefed how AI can improve business operations. Enterprises have been acquiring knowledge from experiences and by utilizing AI. Enterprise Cognitive Computing (ECC) with the help of AI has automated the process of information analysis to speed up the process with accuracy. The capabilities that companies should pose along with the key practices to follow for the organizations to be on the success path were discussed.

Reliable and robust network infrastructure is a challenging task that contributes to enhancing the user experience. The authors in [13] proposed an intelligent framework by utilizing the promising features of SDN and NFV focusing to reduce operational expenses (OPEX). The framework proposed was to fulfil three main requirements being automated network monitoring, autonomic network maintenance, and automated $\&$ dynamic service provisioning. But the proposed framework was not validated using real-time scenarios. The active use of virtualization technologies in organizations automate the management process by constructing modern network infrastructures. The tools used to manage the virtual components dynamically are discussed in [14]. The network infrastructure resources can be efficiently used by automating network equipment management. The authors in [15] have trained the network using an artificial neural network to handle the routing decisions considering the monitoring period. Varying the monitoring period from 3, 5, 10 seconds, various metrics were compared with static, dynamic, and artificial intelligence-enabled routing to prove that the network can learn from past experiences. Machine learning is 
a stream of artificial intelligence, where it is also used to optimize multiple objectives and improve QoS in [16]. Also, this shows that artificial intelligence increases reliable links in the process of communication in terms of TCP and UDP.

\section{DISCUSSION}

\section{A. Trends Impacting $I \& O$}

An important digital transformation has begun leading the enterprises with a massive I\&O transformation. The theme is not about improving the hardware or software but providing delivery of the services to the organizations in an efficient way, matching their needs. The future of I\&O is everywhere, as it is driven by business by nature. Various trends impacting the $\mathrm{I} \& \mathrm{O}$ can be seen from the following fig 1 , which is adopted from [17].

1) Serverless computing: An emerging trend with the capability to provide a function platform as a service (FPaaS) is known as serverless computing [18]. There is no intervention of the user, and the functions to deliver and manage the infrastructure to the user is written as code. Rapid scaling and its granular billing are making FPaaS more exciting and attractive to the users. As the name says, it does not mean that the servers will be eliminated, but it supports utility logic, unpredictable demand, and event-driven requirements. In the coming decade, many organizations will follow this trend. Most of the cloud service providers are moving towards serverless computing by providing the functions abstracted. In a traditional data centre, the abstraction is provided in the physical environment. In contrast, with cloud services like IaaS and PaaS, the abstraction is done for hardware and operating system, respectively. Whereas in serverless computing, the abstraction is done at language runtime, and everything is provided using functions, so it gives function as a Service (FaaS) [19]. Fig 2 shows the user's control over the cloud services. From the figure, it is revealed that serverless computing comes before SaaS because, in SaaS, everything is delivered as software while in serverless, the applications are still in control of the user. Kubernetes is an open-source platform provided by Google for horizontal auto-scaling of applications in the infrastructure, based on resource usage. It is one of the microservice available as a function. The resources, like CPU / RAM, are valuable resources for any organization. Kubernetes implements Horizontal Pod Autoscaler (HPA), a pod is a basic unit of deployment to auto-scale the applications when the load increases. The threshold is fixed at the time of deployment, and the HPA gets triggered once the resource utilization reaches the limit. On the other hand, Amazon web services (AWS) implement the functions using Kinesis and Lambda.

2) Artificial intelligence: AI is a field of computer science, which has been successful in solving many real-time problems like failure recognition, predictive analysis, and others. Also, here AI can be used to perform faster with the growing I\&O requirements, thereby reducing the need for hiring staff. Various streams of AI are used in extracting data, classifying data, clustering, mapping, predicting, and optimizing.

3) Network agility: The changes in the networking field bring automation and orchestration with software-defined networks (SDN), and network function virtualization (NFV) that enables the business to rely on cloud services, IoT, edge services. The network performance affecting parameters like latency, bandwidth utilization, throughput, etc can be varied to make the network more agile.

4) Death of data centre: Realizing the fact that the traditional data centres are consuming a lot of physical resources, several organizations have started to shut down the data centres, and Gartner predicts that by 2025, this may go up to $80 \%$. And the reason for this shutdown is not the technology but the business needs. This trend has put forth a challenge on how to satisfy the customer better by considering the increasing workloads and changing requirements.

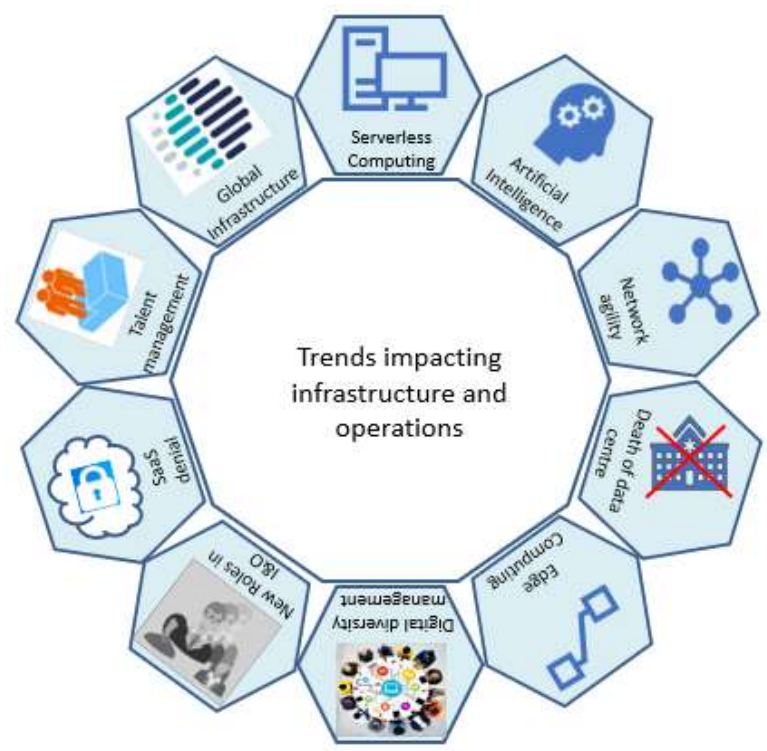

Fig. 1. Trends Impacting I \&O.

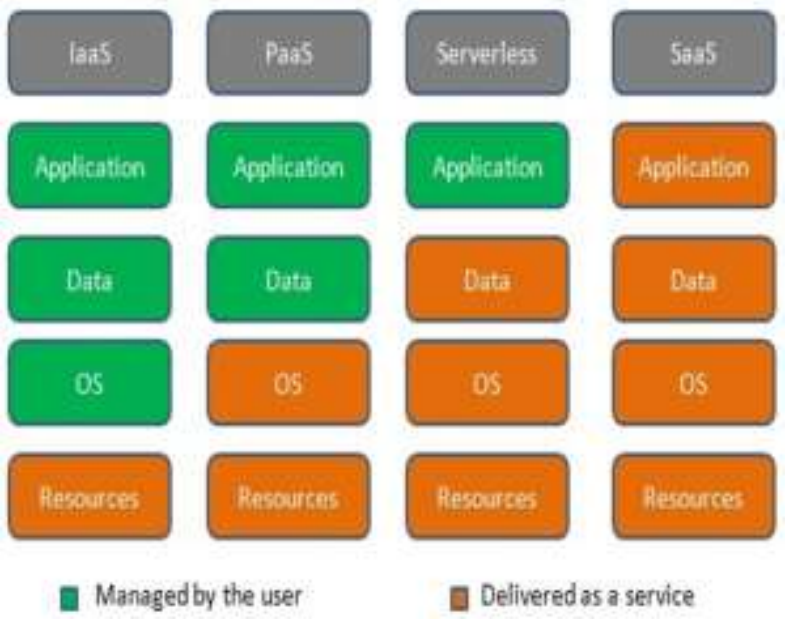

Fig. 2. Control over Cloud Services. 
5) Edge computing: Most of the customers use latencysensitive applications and, in no time, are always ready to shift to other providers if this latency increases. And edge computing is a technology that helps to reduce this latency by considering geographical locations and some laws of physics [20]. Moreover, most of the organizations are moving to industry 4.0, which supports digitization, edge computing helps in attaining better digital quality with increasing demands.

6) Digital diversity management: Diversity, here refers to the heterogeneous resources and different technologies used in digitization by enterprises. The wide use of digital devices in an organization utilizes the resources in distributing and managing digital assets. The management of these diverse resources is a challenge to avoid wastage and maintain accurate information as it directly impacts the business.

7) New roles within $I \& O$ : A significant concern is the lack of skills in maintaining cloud services with the personnel. With the increasing demand for I\&O, the business must target on optimizing the costs and reducing the complexity in supporting new cloud services. The management of cloud services for customizing them according to the needs should be taken up by the new roles.

8) SaaS denial: I\&O is all about maintaining, managing, and providing services to the customers. To better deliver the services with the existing infrastructure, the focus should be put on Software as a service (SaaS) because denial in SaaS may lead to disaster from a security, integrity, and delivery point of view for business.

9) Talent management: Normally, the IT personnel are placed in the organization chart vertically based on the expertise. However, this kind of organization keeps them bounded and restricted as the level moves towards the upward direction, resulting in limited output. Instead, if the IT personnel are horizontally exposed to different technologies, then the success of organizations will have no bounds.

10) Global infrastructure: As the customers access the services from different locations, I\&O requires a global infrastructure to support them. The increase in the scalability of digital access helps the I\&O groups and their supporters to provide infrastructure everywhere. Because the servers are located at different geographical locations, it requires global server load balancing, which extends to L4 and L7 of the Open System Interconnection (OSI) model. The load balancers are gaining significance as many enterprises are moving their applications to the data centres and clouds [21]. Therefore, the Infrastructure and Operations pioneers have come into focus as the trends mentioned above have put forth the challenges and opportunities.

\section{B. Impact of Artificial Intelligence on I \&O}

Infrastructure and Operations is an umbrella with significant sections like helpdesk operations, infrastructure management, and application performance management. The enabler for the transformation in I\&O is the artificial intelligence that interconnects the traditional approaches and new business applications in a highly reliable way. It targets major cost savings, productivity, and performance improvements. It might also lead to the company's market transformation. Artificial intelligence is contributing a majority of spending in $I \& O$ to efficiently utilize the resources. According to International Data Corporation (IDC) ${ }^{1}$, the Worldwide Semiannual Artificial Intelligence Systems Spending Guide expects that the AI systems to reach more than double in spending by 2022. Apart from spending the amount, there is a need for getting the fruit for the amount paid, and so the forecast says that the ground annual growth rate will be around $38 \%$ more during the mentioned period. The artificial intelligence learning process gets the experience from the function performed on the previous collection of input-output pairs and predicts output for upcoming inputs. It creates an environment to improve from the observation of the last contributions. Knowledge and feedback can be used to classify AI learning models.

1) Knowledge-based classification: Based on knowledge, AI learning models can be classified as inductive and deductive learning models. The inductive learning model is based entirely on the input-output pair of data collection. The deductive learning model continues with several regulations and frames new rules to improve efficiency with a particular $\mathrm{AI}$ algorithm.

2) Feedback-based classification: In terms of features, AI learning models can be classified as supervised, semisupervised, unsupervised, and reinforced models based on feedback. Supervised learning models learn from the internal feedback to link the input with output observations. Semisupervised models utilize a collection of compiled, numbered statistics to deduce new labels/attributes from existing databases of information. Semi-supervised models are a safe intermediate between supervised and unsupervised models. Unsupervised models investigate and analyze a sample that does not include any external feedback in attempting to enter statistics. Clustering is a typical case of the unsupervised model. The reinforcement learning model utilizes contrasting mechanisms such as incentives and punishment to "reinforce" special information. This form of mastering method in current AI solutions is increasing significantly. Fig 3 shows how AI is used in various sections of $\mathrm{I} \& \mathrm{O}$. At the helpdesk and customer support where several tickets are handled, the organization can use machine language and natural language processing to handle tickets efficiently. And AI can be used in the infrastructure management section by efficiently managing the resources and predicts the long-term demand considering applications performance.

\footnotetext{
${ }^{1}$ https://www.idc.com/getdoc.jsp?containerId=IDC_P33198.
} 


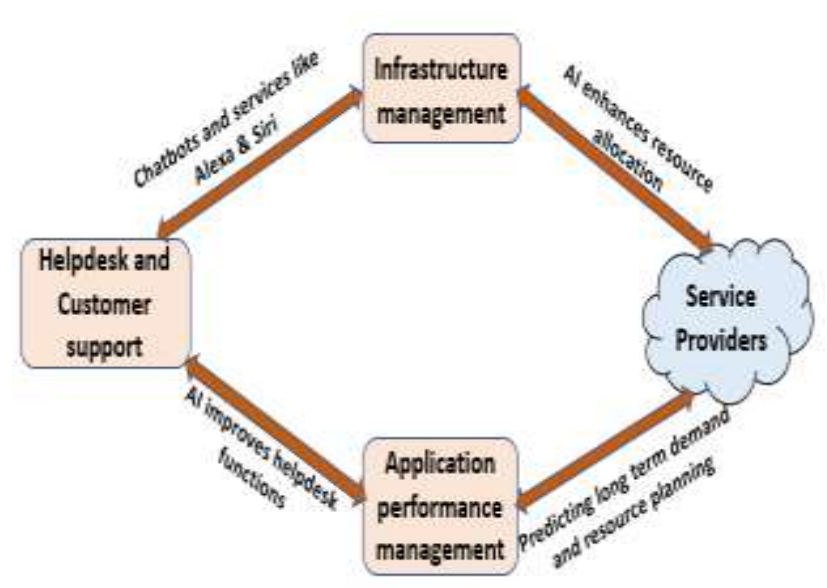

Fig. 3. AI-Driven Infrastructure and Operations.

Artificial intelligence is often debated, but many significant achievements are still in progress [22]. The most significant headwind of $\mathrm{AI}$ is its investment costs, which in the short term will skew returns. Nevertheless, if the change occurs, companies that make the investments should expect a significant performance gap to be unfairly rewarded. The AI techniques must address another essential element that every strategy needs: a code of ethics. Humans have developed artificial intelligence, and so it is not neutral and can be exposed to be biased [23]. Instances of such bias are already found in image searches, financial searches, etc. The technology providers must follow certain principles as shown in the following Table I, to ensure that AI is free of bias.

AI algorithms are readily available in the open-source environment or via cloud-based APIs, using a vast machine learning (ML) network [24]. Therefore, the availability of an AI algorithm designed for customization, optimization, search, or recommendation improves the customer experience by enhancing the quality of service (QoS). However, the commoditized AI algorithm lacks the following which is mentioned in Table II to match the user's requirements.

TABLE I. PRINCIPLES TO BE FOLLOWED BY TECHNOLOGY PROVIDERS

\begin{tabular}{|l|l|}
\hline Principle & Explanation \\
\hline Utility & $\begin{array}{l}\text { Ensure that the AI algorithms are clear, useful, and } \\
\text { delightful for the user by using holistic metrics as } \\
\text { the aim is not only to generate revenue but also to } \\
\text { focus on social outcomes. }\end{array}$ \\
\hline Empathy and respect & $\begin{array}{l}\text { Evaluate the AI algorithms by satisfying the } \\
\text { implicit and explicit needs of the population } \\
\text { without having homogenous teams in the } \\
\text { community and consider diversity. }\end{array}$ \\
\hline Trust & $\begin{array}{l}\text { Gaining trust is essential by being open, secure, } \\
\text { and stable in behaviour. }\end{array}$ \\
\hline Fairness and safety & $\begin{array}{l}\text { In the real world, where these algorithms are used } \\
\text { should not cause any harm either physically or } \\
\text { digitally. }\end{array}$ \\
\hline Accountability & $\begin{array}{l}\text { A degree of responsibility must be set by } \\
\text { measuring the performance and restructure it if the } \\
\text { customers are unsatisfied. }\end{array}$ \\
\hline
\end{tabular}

TABLE II. DRAWBACKS OF COMMODITIZED AI ALGORITHMS

\begin{tabular}{|l|l|}
\hline Drawback & Description \\
\hline $\begin{array}{l}\text { Lacks } \\
\text { matching } \\
\text { requirements }\end{array}$ & $\begin{array}{l}\text { The truth is that commoditized algorithms are not readily } \\
\text { available for competition. AI is just a commoditized tool } \\
\text { without human intelligence to change algorithms. Humans } \\
\text { must, therefore, incorporate technology to program } \\
\text { algorithms as per requirements. }\end{array}$ \\
\hline $\begin{array}{l}\text { Human } \\
\text { ontelligence } \\
\text { strengthens AI }\end{array}$ & $\begin{array}{l}\text { Many technology firms are racing in the context of the AI } \\
\text { revolution to harness AI, ML, and big data and drive } \\
\text { further functionality and customization into individual } \\
\text { client experiences. They can not only bear in mind the } \\
\text { current needs of users but also function constructively to } \\
\text { predict their customer's tastes, moods, desires, and issues. }\end{array}$ \\
\hline $\begin{array}{l}\text { Lack of } \\
\text { efficacy }\end{array}$ & $\begin{array}{l}\text { The commoditized AI algorithms lack efficacy as the } \\
\text { results generated may not match with the expected results. }\end{array}$ \\
\hline
\end{tabular}

The available commoditized AI algorithm needs to be seamlessly blended to match the requirements. The following framework should be followed in customizing the algorithms

- Step 1: Understand and define the goals you want your AI to achieve. This is achieved by introducing programmatic or visual methods for software-taking IT teams to see how the AI works in its present state.

- Step 2: The second stage is the implementation of the highest integrity criteria for the AI. IT managers and programmers should be able to explain what the AI is doing to personalize the AI. To understand what works and what is not in the algorithm, analytics is an integral part of the equation.

- Step 3: Then, program your intellectual property to map your specifications for the algorithms.

This is the best way to maximize the competitive edge that any algorithmic output can achieve and see if it aligns with or does not meet requirements.

The new algorithms proposed using AI technology use pattern matching techniques and implement them in the current scenario by customizing them according to the requirements. An unskilled person will ideally be able to focus on actual business outcomes through editing or augmenting the outputs in these AI algorithms sometime within the nottoo-distant future and will not have to rely on the technical person to do so. This seamless combination of people and machines requires trust from above, which can be accomplished if proof and validations that their algorithm modification works are collected. Every day, algorithms get smarter, but alone somehow cannot do it. As the algorithms evolve, people are seeking to sharpen their intellect, to link the points, and to push towards a smarter future for business and beyond.

\section{AI-enabled SDN in $I \& O$}

AI-enabled SDN helps the cloud companies to offer superior user interface application efficiency. The SDN enables application-conscious routing through the network via intelligence and the identification of apps. The required QoS and security policy enforcement is given to each class of requests, according to business requirements. Dynamism in resource distribution is an inevitable necessity to resolve the 
issues in the I\&O of any organization [25]. In other words, network services are delegated on request and released immediately after use. The various sections in I\&O communicate and share the data through essential networking techniques. Rather when these sections communicate using AI-enabled SDN as shown in fig 4, then the resources can be intelligently used by improving the performance. AI-enabled SDN optimizes the distribution of requests across all servers to improve the QoS.

At present, SDN is the most appealing facilitator for the deployment of dynamic resources. The various streams of AI can be applied in SDN to optimize the performance in I\&O. Using the inductive learning model, the managers can better extract the desired output as the inputs provided can be controlled. From the experiences of past behaviour, new rules can be discovered by implementing this model. The network administrators have centralized control over all the network operations. The deductive learning model is instructionoriented learning. The concepts and rules are induced to all employees in the organization by providing training. The SDN controller's global perspective of the usage of network services for all the participating users is part of the infrastructure sharing [26]. The controller functions as a broker between the tenants and the network owner and communicates each tenant to network service functionality (e.g. network use status) securely via authentication/authorization. This enables service trackers to dynamically ask the service manager for network support and agreements for SLAs through signals. Conventional networking is no longer appropriate mostly because of the rear-mounting of all traffic from branches to headquartersincluding that for the cloud-contributes to latency and affects application efficiency. SDN offers network simplification, reduced costs, productivity in bandwidth, and seamless cloudbased on-ramps with substantial application performance, particularly for critical applications, with no privacy or protection. The works discussed in [15] and [16] are evidence of how artificial intelligence fusion with SDN has improved the results in terms of performance.

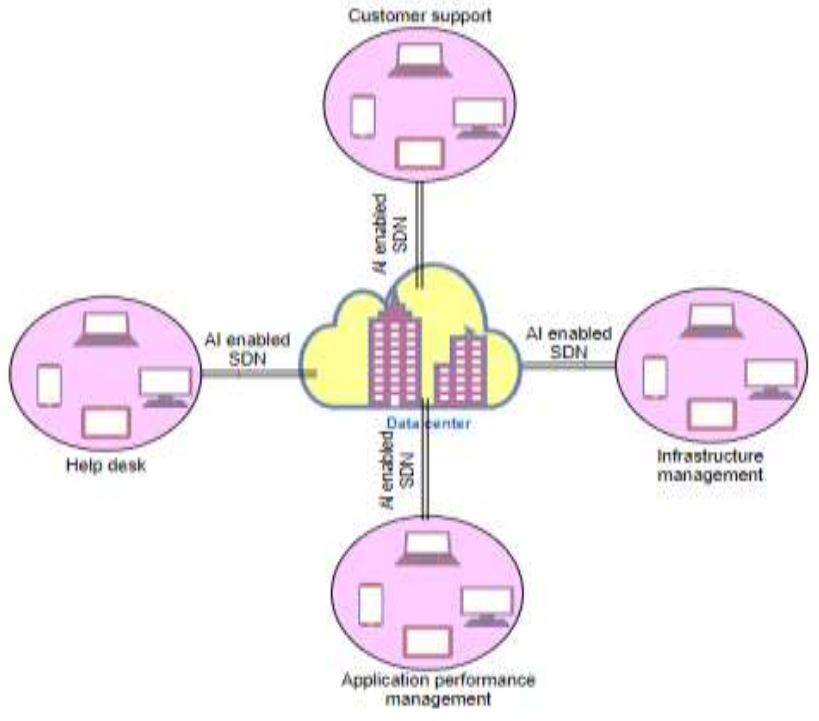

Fig. 4. AI-enabled SDN in Infrastructure and Operations.
1) Combining $S D N$ with 5G: $5 \mathrm{G}$ networks spread across vertical and regional areas over the next decade are increasing. The service providers may provide the services at an increased bandwidth and minimize latency with a price costing less per bit than today. The parallel adoption of 5G and SD-WAN [27] makes it essential that organizations understand what these technologies can do for companies.:

- To achieve the agility, costs, and flexibility necessary for the digital age, all techniques are guided and applied with a disassembled model that separates hardware and software, and the data plane from the control plane.

- In deployments for improved customer experience, both technologies will be distributed. Organizations will offer completely new CX technologies such as AR / VR in retail.

- 5G can provide a high-performance connectivity underlay network that can be made more widely available in many areas where the high-speed broadband service wireline networks cannot deliver [28]. The integration of $5 \mathrm{G}$ in SDN improves network performance in terms of throughput, latency, energy efficiency, connectivity, service deployment, reliability, etc.

\section{Benefits of AI-enabled SDN in I \&O}

- Efficient: The efficiency in terms of provisioning time is indisputable. It shortens the provisioning cycle from seconds to milliseconds and this significant reduction, in turn, has increased the efficiency.

- Automated: The integration of AI has enabled the process to be automated. The functions to be performed are triggered timely based on the actions programmed and does not need any human intervention.

- Reduced human errors: Manual operations result in misconduct of operations by making errors in network misconfiguration. But the automation reduces such errors.

- Secure: The transmission of data using AI-enabled SDN is secure. As it gives a centralized view of the whole network and easily detects anomalies and attacks.

- Consistent: A key benefit of AI-enabled SDN is to efficiently use various modes of network transport. A simple solution is to block the traffic coming from an underperforming link and redirect it to a better performing link to ensure consistency in providing the services.

- Monitoring and better management: An AI-enabled SDN intelligently monitors and manages to support the transport services. It improves network performance by enhancing the metrics used to measure the QoS (packet loss rate, latency, jitter, throughput).

- Zero-touch provisioning: The centralized orchestration and control over the network enable to configure the devices automatically without the intervention of the network administrator. 
- Storage management: The storage resources can be optimally utilized by performing predictive analysis and the storage space can be adjusted based on necessity.

\section{Challenges AND Future Directions}

The following are the challenges faced by the organizations in the process of transforming from traditional networking to AI-enabled SDN from the I\&O perspective.

- Deployment: Wi-Fi is today the most common connectivity used in the IT sector. SD-WAN plays a significant role in transforming the edge network in IT infrastructure, but the question arises on how sensors and devices are deployed and linked, which are so prevalent within production companies. The challenges concerned with coverage, intrusion, capability, and security are unaddressed.

- Threat Detection and Analysis: Several solutions are available in new generations of wireless internet access points to deal with this problem by extending the network or radio technology available. However, safety is the main challenge because establishing a safe and secure network is crucial to the operation of businesscritical IT infrastructure along with managing them within the manufacturing company. Prevention of outages and disruptions is a major concern. The drastic changes in technology though it brings advancement yet comes with various open threats that have to be handled.

- Protection: Protection has increasingly been the focus when regulatory authorities and end-users want to have a free and fair exchange. It can be provided using a heuristic-based approach and intelligence-driven as protection in providing services requires tight integration at the system level. Many companies create AI advice engines so reliable that very little information is required about the individual who makes a choice, and that customization is a challenge to be addressed.

- Resource utilization: Another challenge concerned with the infrastructure is the communication between the representative and clients that consumes a lot of resources, time, and cost. So to minimize this, the AI technology system comprises of specialized software for a pre-check site and argument sheet, where the Automatic speech recognizing (ASR), converts audio into text, and by using natural language processing (NLP) the researchers have taken the task of reducing the consumption of resources and improving the performance.

- Next-generation implementation: With 5G implementations in the industry becoming more popular, $5 \mathrm{G}$ will provide an additional or alternative Wi-Fi solution. Based on various analogies, the key difference between $5 \mathrm{G}$ and $\mathrm{Wi}-\mathrm{Fi}$ is with its implementation and management in terms of range and power consumption. Through 5G, a company can connect its sensors and devices through the network in a fully managed and secure environment. Globally, mobile network providers aim to deliver $5 \mathrm{G}$ services operated to businesses in precisely such an area. The full implementation of this next-generation networking has many new challenges unaddressed.

- Cyber-security: In the present situation of the pandemic, most of the employees are performing their tasks by work from home using different networks. This has made it the attackers easy to penetrate the network. Most of the organizations are investing now in making this more secure and is an open area of research.

\section{CONCLUSION}

Infrastructure and Operations in any organization need to be efficient to benefit its stakeholders. The transformation of communication mode from traditional to SDN in various sections of I\&O gives a centralized control. AI's role in the field of networking with a capacity to program the network in SDN has changed the perception of providers and users. Various trends impacting I\&O are discussed with a focus on AI-enabled SDN in I\&O. The softwarization of the networking provides the users with applications, data, and resources along with a platform to use them. This research contributes to putting forward a framework following a stepwise process of converting commoditized AI algorithms to customized algorithms matching the requirements. Also, the impact of AI on various sections of I\&O highlights the benefits of integrating AI-enabled SDN in I\&O. The challenges discussed in the process of implementing AIenabled SDN, from the infrastructure and operations perspective put forward future directions for the researchers to explore more in the area.

\section{REFERENCES}

[1] L. Agostini and R. Filippini, "Organizational and managerial challenges in the path toward Industry 4.0," European Journal of Innovation Management, vol. 22, no. 3, pp. 406-421, 2019.

[2] N. Javaid, A. Sher, H. Nasir, and N. Guizani, "Intelligence in IoT-based 5G networks: Opportunities and challenges," IEEE Communications Magazine, vol. 56, no. 10, pp. 94-100, 2018.

[3] Q.-V. Pham et al., "A survey of multi-access edge computing in 5G and beyond: Fundamentals, technology integration, and state-of-the-art," IEEE Access, vol. 8, pp. 116974-117017, 2020.

[4] A. A. Barakabitze, A. Ahmad, R. Mijumbi, and A. Hines, "5G network slicing using SDN and NFV: A survey of taxonomy, architectures, and future challenges," Computer Networks, vol. 167, p. 106984, 2020.

[5] D. Kim, Y.-H. Kim, C. Park, and K.-I. Kim, "KREONET-S: Softwaredefined wide area network design and deployment on KREONET," IAENG International Journal of Computer Science, vol. 45, no. 1, pp. 27-33, 2018.

[6] K. Zkik, G. Orhanou, and S. El Hajji, "Secure mobile multi-cloud architecture for authentication and data storage," International Journal of Cloud Applications and Computing (IJCAC), vol. 7, no. 2, pp. 62-76, 2017.

[7] R. Buyya et al., "A manifesto for future generation cloud computing: Research directions for the next decade," ACM computing surveys (CSUR), vol. 51, no. 5, pp. 1-38, 2018.

[8] G. Zhang, T. Li, Y. Li, P. Hui, and D. Jin, "Blockchain-based data sharing system for ai-powered network operations," Journal of Communications and Information Networks, vol. 3, no. 3, pp. 1-8, 2018.

[9] E. Karaaslan, U. Bagci, and F. N. Catbas, "Artificial intelligence assisted infrastructure assessment using mixed reality systems," Transportation Research Record, vol. 2673, no. 12, pp. 413-424, 2019.

[10] P. Grover, A. K. Kar, and Y. K. Dwivedi, "Understanding artificial intelligence adoption in operations management: insights from the 
review of academic literature and social media discussions," Annals of Operations Research, pp. 1-37, 2020.

[11] S. Chanias, M. D. Myers, and T. Hess, "Digital transformation strategy making in pre-digital organizations: The case of a financial services provider," The Journal of Strategic Information Systems, vol. 28, no. 1, pp. 17-33, 2019.

[12] M. Tarafdar, C. M. Beath, and J. W. Ross, "Using AI to enhance business operations," MIT Sloan Management Review, vol. 11, 2019.

[13] P. Neves et al., "Future mode of operations for 5G-The SELFNET approach enabled by SDN/NFV," Computer Standards \& Interfaces, vol. 54, pp. 229-246, 2017.

[14] A. Klimova, S. Kodolov, K. Aksyonov, and A. Y. Filimonov, "Implementing Dynamic Management Of Virtual Network Infrastructure Components," in CEUR Workshop Proceedings, 2019, vol. 2525: CEUR-WS.

[15] Wu, Y. J., Hwang, P. C., Hwang, W. S., \& Cheng, M. H., “Artificial Intelligence Enabled Routing in Software Defined Networking." Applied Sciences, vol. 10 no.18, 6564, 2020.

[16] A. Akbar, M. Ibrar, M. A. Jan, A. K. Bashir, and L. Wang, "SDNenabled Adaptive and Reliable Communication in IoT-Fog Environment Using Machine Learning and Multi-Objective Optimization," in IEEE Internet of Things Journal, doi: 10.1109/JIOT.2020.3038768.

[17] Gartner, "Gartner IT Infrastructure, Operations \& Cloud Strategies Conference," $2019 . \quad$ [Online]. Available: https://www.gartner.com/en/newsroom/press-releases/2019-09-09gartner-announces-it-infrastructure-operations-and-cloud-strategiesconference-2019.

[18] P. Castro, V. Ishakian, V. Muthusamy, and A. Slominski, "The rise of serverless computing," Communications of the ACM, vol. 62, no. 12, pp. 44-54, 2019.
[19] A. Dakkak, C. Li, S. G. De Gonzalo, J. Xiong, and W.-m. Hwu, "Trims: Transparent and isolated model sharing for low latency deep learning inference in function-as-a-service," in 2019 IEEE 12th International Conference on Cloud Computing (CLOUD), 2019: IEEE, pp. 372-382.

[20] H. Luo, H. Cai, H. Yu, Y. Sun, Z. Bi, and L. Jiang, "A short-term energy prediction system based on edge computing for smart city," Future Generation Computer Systems, vol. 101, pp. 444-457, 2019.

[21] M. R. Belgaum et al., "A behavioral study of task scheduling algorithms in cloud computing," International Journal of Advanced Computer Science and Applications, vol. 10, no. 7, pp. 498-503, 2019.

[22] R. Vinuesa et al., "The role of artificial intelligence in achieving the Sustainable Development Goals," Nature Communications, vol. 11, no. 1, pp. 1-10, 2020.

[23] E. Ntoutsi et al., "Bias in data-driven artificial intelligence systems-An introductory survey," Wiley Interdisciplinary Reviews: Data Mining and Knowledge Discovery, vol. 10, no. 3, p. e1356, 2020.

[24] N. Gift, Pragmatic AI: An Introduction to Cloud-based Machine Learning. Addison-Wesley Professional, 2018.

[25] Chien, W.-C., C.-F. Lai, and H.-C. Chao, Dynamic resource prediction and allocation in C-RAN with edge artificial intelligence. IEEE Transactions on Industrial Informatics, 2019. 15(7): p. 4306-4314.

[26] Chaudhary, R., et al., BEST: Blockchain-based secure energy trading in SDN-enabled intelligent transportation system. Computers \& Security, 2019. 85: p. 288-299.

[27] Z. Duliński, R. Stankiewicz, G. Rzym, and P. Wydrych, "Dynamic Traffic Management for SD-WAN Inter-Cloud Communication," IEEE Journal on Selected Areas in Communications, 2020.

[28] Prerna, D., R. Tekchandani, and N. Kumar, Device-to-device content caching techniques in 5G: A taxonomy, solutions, and challenges. Computer Communications, 2020. 153: p. 48-84. 